\title{
Skipping breakfast is associated with nutrient gaps and poorer diet quality among adults in the United States
}

\author{
S. Fanelli ${ }^{1}$, C. Walls ${ }^{2}$ and C. Taylor ${ }^{3}$ \\ ${ }^{1}$ Doctoral Student, College of Medicine, The Ohio State University, Columbus, Ohio, \\ ${ }^{2}$ Masters Student, College of Medicine, The Ohio State University, Columbus, Ohio and \\ ${ }^{3}$ Professor, College of Medicine, The Ohio State University, Columbus, Ohio, 43210, US
}

Habitual breakfast consumption is associated with decreased risk for chronic disease as well as greater micronutrient intakes, especially folate, calcium, iron, phosphorus, and vitamins A, B1, B2, B3, C, and D (1). Although breakfast is prioritized for children, many adults skip breakfast on a frequent basis (2). Suboptimal intakes of such micronutrients may exacerbate chronic disease development (3). Therefore, this study aimed to explore the differences in energy and nutrient intakes as well as diet quality across all meals for adults who consume versus skip breakfast. We hypothesized that adults who consume breakfast have better diet quality than breakfast skippers.

Data were examined from 30,889 adults, 19 years or older, from the 2005-2016 National Health and Nutrition Examination Survey (NHANES). Participants were non-pregnant, non-institutionalized US citizens. The 24-hour dietary recall using the validated automated multiple-pass method was used to record all intakes from midnight to midnight from the previous day. Recall data were transformed into nutrient estimates and MyPlate equivalents using the Food and Nutrient Database for Dietary Studies and Food Patterns Equivalents Database, respectively. Participants self-designated eating occasions (breakfast, lunch, dinner, snacks). Dietary intakes were aggregated across eating occasions to estimate intakes from each meal. Adults were classified as breakfast skippers if they did not report food or beverage intake at breakfast during the recall. Micronutrient intakes for the total day were compared to the Estimated Average Requirement (EAR) or Adequate Intake (AI). The Healthy Eating Index-2015 was used to estimate diet quality compared to the Dietary Guidelines for Americans. Mean differences were compared using analysis of covariance, controlled for age, sex, income, race and ethnicity, and marital status. CDC weighting schemes were applied to produce nationally representative estimates of nutritional intakes.

Breakfast skippers consumed significantly more energy (kcal), carbohydrates, total and saturated fat, and added sugars during lunch, dinner and snacks than those who consumed breakfast on the day of intake $(\mathrm{p}<0.001)$. Adults who skipped breakfast had lower total intakes and were significantly less likely to meet the EAR or AI for folate, calcium, iron, vitamins A, B1, B2, B3, C, and $\mathrm{D}$ on the day of intake $(\mathrm{p}<0.001)$. Adults who skipped breakfast had significantly poorer overall diet quality for the day of intake than those who consumed breakfast $(\mathrm{p}<0.001)$.

Adults who skipped breakfast consumed less folate, calcium, iron, and select vitamins for the total day, and those nutrients commonly consumed at breakfast were not made up later in the day from other meals on the day of record. Additionally, those who skipped breakfast had significantly poorer overall diet quality than those who ate breakfast. Breakfast provides a unique opportunity to consume important micronutrients that may be less present in subsequent meals.

\section{Acknowledgments}

This project was supported by a grant from the American Dairy Association Mideast

\section{References}

1. Deshmukh-Taskar P, Radcliffe J, et al. (2010) J Am Coll Nutr 29, 407-18.

2. Gibney M, Barr S, et al. (2018) Nutrients 10, 559.

3. Uzhova I, Mullally D, et al. (2018) Nutrients 10, 1578.

4. Iqbal K, Schwingshackl L, et al. (2017) Eur J Clin Nutr 71, 1312-20. 